\title{
OPTIMIZATION OF HEAT TREATMENT OF CASTINGS
}

\author{
DUCHEK, M[ichal]; TIKAL, F[ilip] \& BOZIK, M[artinek]
}

\begin{abstract}
The use of various types of software for rapid identification of potential problems in manufacturing processes is becoming ever more popular. One of such programs is DEFORM, simulation software for forming and heat treatment processes. The purpose of this study was to construct a $3 D$ model of a specific casting, to identify its critical locations and then select the optimum heat treatment procedure preventing cracking. Results of this work include a detailed analysis of stress and temperature fields in the cast part.
\end{abstract}

Key words: numerical simulation, heat treatment, casting

\section{INTRODUCTION}

Inadequate heat treatment may cause significant problems and often irreversible changes in the part, leading to considerable financial costs. Companies have therefore begun making use of the benefits of numerical simulation. Simulation of the entire manufacturing process is a relatively easy and accurate method for revealing critical locations in models of intricate shape. It also enables heat treatment procedures to be optimized to avoid large stresses which might initiate cracks.

\section{HEAT TREATMENT}

The company PILSEN STEEL manufactures complex castings, in which casting defects often need to be removed by welding (Hosnedl et al., 2010). Such castings are then heat treated to relieve stresses introduced by welding. The heat treatment of complex-shape castings should be designed very carefully in order to prevent additional distortion of the part (Levíček \& Stránský, 1984).

Finding the locations of critical stresses may also aid in adapting the mould for the entire casting. A modification to the geometry of the casting may eliminate such critical locations (Tikal \& Urbánek, 2010).

The casting in question is shown in Fig. 1.

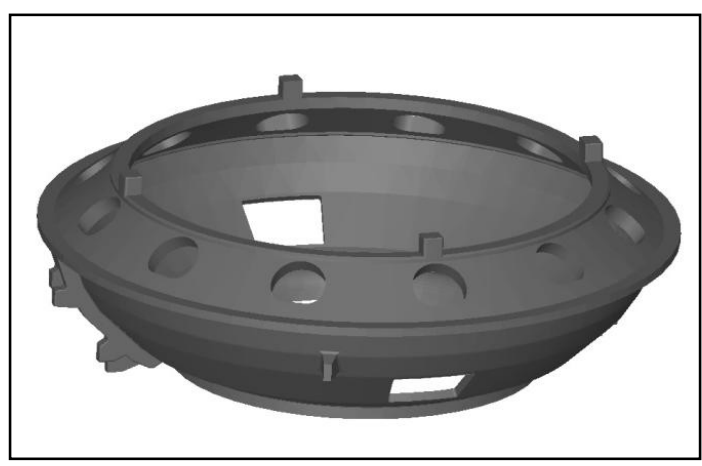

Fig. 1. CAD model "UFO1" modified with the aid SolidWorks software for the purpose of simulation
The full schedule of the heat treatment is shown in Fig. 2. Heating to $250^{\circ} \mathrm{C}$ was the first stage. Repair welding of the casting defects was followed by cooling to $100^{\circ} \mathrm{C}$. The following stress relieving comprised slow heating to $740^{\circ} \mathrm{C}$, an 8-hour hold and controlled cooling in furnace down to $200^{\circ} \mathrm{C}$. The casting finally cooled down in air.

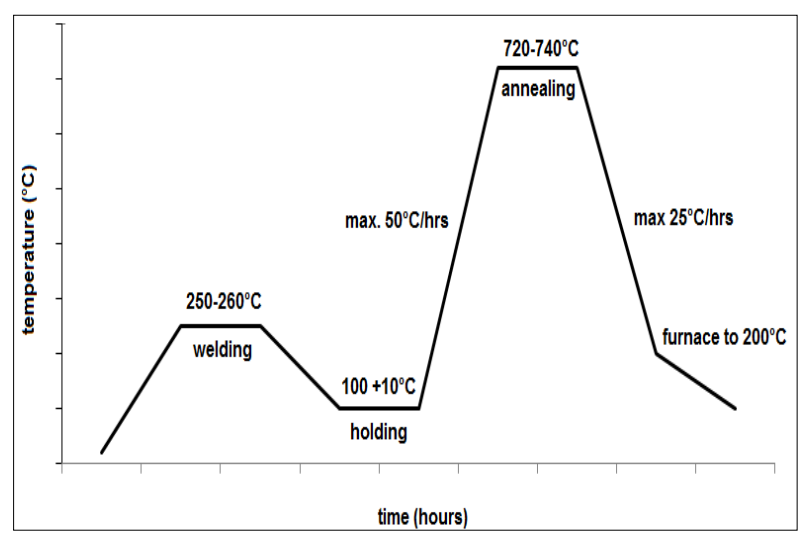

Fig. 2. Heat treatment schedule

\section{NUMERICAL SIMULATION}

The above heat treatment process was simulated using the software DEFORM. The capabilities of this FEM system include modelling of static load applications and effects of large strains in hot and cold forming processes (J.G. Yang et al., 2004). It provides predictions of temperature, strain and stress distributions for each time instant of the process. The primary focus of the simulation was the heat treatment after welding, i.e. stress relieving (Jinwu Kang \& Yiming Rong, 2005).

The input model was supplied by PILSEN STEEL s.r.o. It is a CAD model of UFO1 casting after machine cleaning and redressing and before removing defect and heat treating. The casting has been fitted together (its halves have been joined with clamps).

Before the actual simulation, the model had to be modified in the CAD software SolidWorks (Fehler! Verweisquelle konnte nicht gefunden werden.). After simplifying the casting geometry, a numerical model of the heat treatment process was constructed in DEFORM 3D. The time scale units of the heat treatment process were converted to seconds for use by the simulation software (Fig. ).

The numerical simulation was refined and updated to reflect the real-world conditions as exactly as possible. Stresses introduced by welding were idealized through rapid heating of the entire casting to $120^{\circ} \mathrm{C}$ and cooling down to $100^{\circ} \mathrm{C}$. The purpose of the simulation was to explore the stress state in UFO1 casting during heat treatment. 


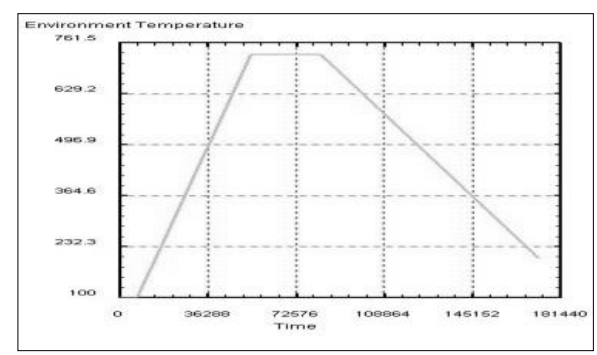

Fig. 3. Heat treatment schedule after heat treatment shown with the horizontal axis in seconds

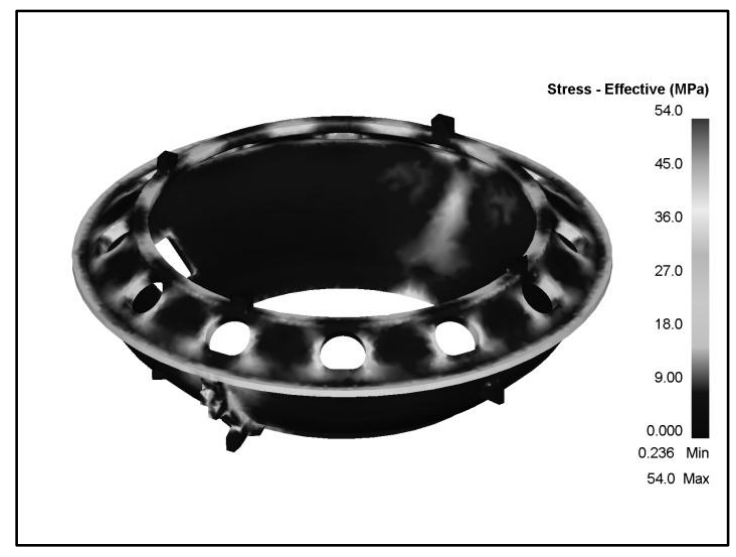

Fig. 4. Distribution of stress in the casting

Fig. shows the distribution of stress in the casting at the time of its maximum. This is the maximum for the entire duration of the heat treatment.

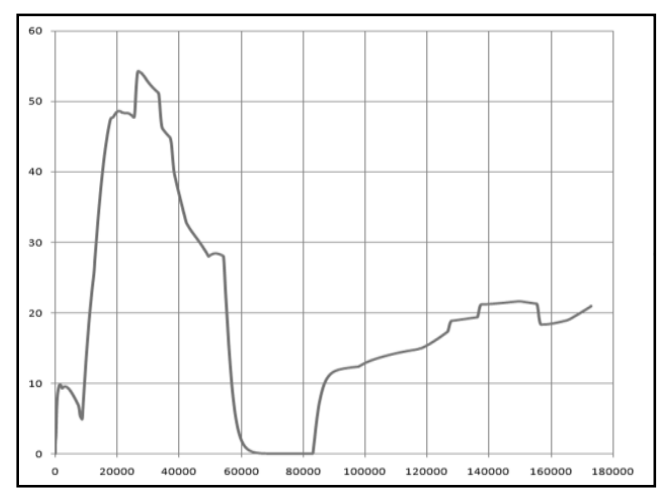

Fig. 5. Stress in the casting vs. time

Fig. 5 is a detailed representation of maximum stress values vs. time in seconds.

The maximum stress shown occurs at the marked time instant $\mathrm{t}=27,900$ s, i.e. $7.75 \mathrm{hrs}$.

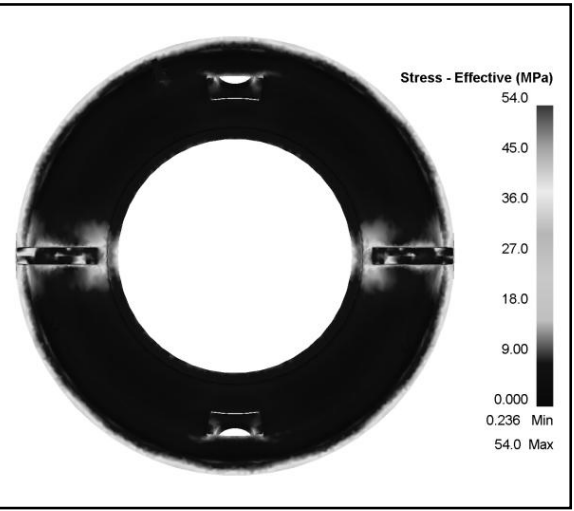

Fig. 6. Stress distribution on the bottom side of the casting
Fig. 6 shows the distribution of stress in the bottom part of the casting at the time of its maximum.

The most heavily stressed locations include the top and middle flanges, areas around the "windows" and two large protrusions on the sides of the casting.

Numerical simulations were performed for multiple schedules. Stress behaviour and magnitude vs. time and vs. heat treatment temperature were tracked. Approx. 20\% temperature variation does not dramatically change the resulting stress values. The duration of the stress remains similar as well. This is why the above schedule is deemed the optimal one.

\section{CONCLUSION}

The numerical simulation accounted for the stress introduced by welding, to some extent, but as the stress was impossible to define precisely in terms of magnitude and location, the result is mostly theoretical. Despite this, the simulation yielded a detailed analysis of the stress relieving process in the casting. The results revealed the square openings, the flange end and the protrusions on the casting sides as the weak spots. The overall model was then adjusted with respect to these critical locations and successfully reduced the risk of cracking, taking account of the potential repair welding operations.

The following stage of the project will improve the accuracy of the numerical model through a more detailed description of the stress introduced during welding before heat treatment. In addition, two other geometries will be simulated: UFO2 and UFO3, which require calculations of similar heat treatment procedures. Two schedules will be explored for each geometry. Risers will be removed by flame cutting at different points of each schedule. For this reason, the model will have to be changed during simulation from one with risers to one without them. In this case, values of the individual calculated quantities up to riser cutting will have to be transferred to the model without risers and the simulation of heat treatment will be resumed. Parameters tracked in UFO2 and UFO3 models will be the same as those in the UFO1 model. The purpose will be to find a more suitable heat treatment schedule.

\section{ACKNOWLEDGEMENTS}

This paper includes results achieved within the project FRTI1/492 - Use of Advanced Medium and High-Alloyed Steels in Power Industry.

\section{REFERENCES}

Hosnedl, P., Nový, Z., Konopík, P., Džugan, J. \& Tikal, F., (2010). Structural materials and fluoride salts for high temperature applications, Plzeň, COMAT 2010, ISBN 97880-254-8683-2

Levíček, P. \& Stránský, K. (1984). Metalurgické vady ocelových odlitki̊, SNTL - Nakladatelství technické literatury, 04-415-84

Tikal, F. \& Urbánek, M. (2010). Simulace sálání v peci v programu MSC.Marc/Mentat, Brno, Setkani uzivatelu MSC.Software s.r.o., From 2002

J.G. Yang, J.S. Gao, H.Y. Fang, G.T. Zhou J.F. Hu (2007).Simplified Heat Model of the Weaving Welding Processes, the 5th International Conference on Physical and Numerical Simulation of Materials Processing, China

Jinwu Kang \& Yiming Rong (2005). Modeling and simulation of load heating in heat treatment furnaces, Beijing MA 01609, China 